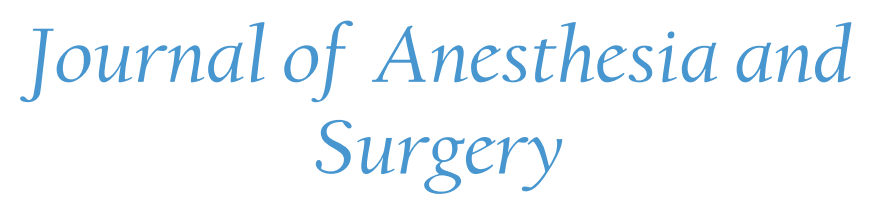

\title{
Continuous Spinal Anaesthesia (CSA) for Emergency Laparotomy in High-Risk Elderly Patients: Technique and Outcomes of a Prospective Service Evaluation
}

\author{
Siti H.M.A Basar ${ }^{1}$, Chinthaka Warusawitharana ${ }^{2}$, Siby Sebastian $^{3}$, Elda Camacho ${ }^{3}$, G. Niraj ${ }^{*}$ \\ ${ }^{1}$ Specialist Trainee in Anaesthesia, Department of Anaesthesia, Leicester General Hospital, University Hospitals of Leicester NHS \\ Trust, Gwendolen Road, Leicester, LE5 4PW, UK \\ ${ }^{2}$ Speciality Doctor in Anaesthesia, Department of Anaesthesia, Leicester General Hospital, University Hospitals of Leicester NHS \\ Trust, Gwendolen Road, Leicester, LE5 4PW, UK \\ ${ }^{3}$ Consultant in Anaesthesia, Department of Anaesthesia, Leicester General Hospital, University Hospitals of Leicester NHS Trust, \\ Gwendolen Road, Leicester, LE5 4PW, UK
}

"Corresponding author: Dr. G. Niraj, Consultant in Anaesthesia \& Pain Medicine, Clinical Research Unit in Pain Medicine, University Hospitals of Leicester NHS Trust, Gwendolen Road, Leicester, LE5 4PW, Tel: 0044116258 4661;

Email: nirajgopinath@yahoo.co.uk

\begin{abstract}
Continuous Spinal Anaesthesia is a recognized technique for providing anaesthesia for various surgical procedures. It may be an alternative to general anaesthesia in high-risk elderly patients requiring emergency laparotomy. The objective was to evaluate the benefits of continuous spinal anaesthesia in providing effective anaesthesia for emergency laparotomy, in enhancing recovery after major abdominal surgery and in reducing length of stay in the intensive care unit in high-risk elderly patients. Prospective service evaluation was performed at a tertiary care university hospital. High-risk elderly patients were offered both general anaesthesia and continuous spinal anaesthesia. An 18-gauge macro catheter was inserted into the intrathecal space through a 16-gauge Tuohy needle. Sedation was maintained with remifentanil. Prophylactic anti-emetics were administered. Blood pressure was maintained with an infusion of metaraminol. Over a 27 -month period, 25 high risk elderly patients were offered continuous spinal anaesthesia and general anaesthesia for emergency laparotomy. Twenty-one patients opted for continuous spinal anaesthesia. Three patients required conversion to general anaesthesia. In the remaining 18 patients, continuous spinal anaesthesia provided adequate anaesthesia for major bowel surgery. Mean length of stay in level 2 care was 1.6 days. 30 -day mortality was $14 \%$. None of the patients reported post dural puncture headache. There were no neurological complications.

Emergency laparotomy carries well-recognised intra and postoperative risks and in the elderly cohort, these risks are significant and probably life threatening. Continuous spinal anaesthesia is a well-established technique that may offer some reduction of both intra and postoperative risks with an improved immediate recovery from major emergency surgery.
\end{abstract}

Received date: October 10, 2017 Accepted date: November 10, 2017 Published date: November 14, 2017

Citation: Niraj, G., et.al. Continuous Spinal Anaesthesia (CSA) for Emergency Laparotomy in High-Risk Elderly Patients: Technique and Outcomes of a Prospective Service Evaluation. (2017) J Anesth Surg 4(2): 130- 133.

\section{DOI: $10.15436 / 2377-1364.17 .097$}

Keywords: Continuous spinal anaesthesia; Emergency laparotomy; Elderly patients

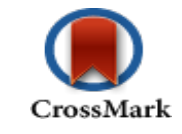

\section{Introduction}

Continuous Spinal Anaesthesia (CSA) is a recognized technique for providing anaesthesia for various surgical procedures including lower limb surgery, vascular surgery, urological procedures and elective laparotomy ${ }^{[1-3]}$. However it has remained an underutilized technique in the modern anaesthesia practice. CSA utilizes incremental dosing of an intrathecal local anaesthetic for an indefinite duration, allowing titration of the block level to the surgical requirement with greatly improve haemodynamic con- 
trol when compared to other neuraxial techniques ${ }^{[2-4]}$. It can be a useful alternative to general anaesthesia in high-risk elderly patients requiring emergency laparotomy. We report a prospective case series of high-risk elderly patients who underwent CSA for emergency laparotomy and detail the technique and its various benefits. We present an argument for an increased application of CSA for emergency laparotomy in this cohort.

\section{Methods}

High-risk elderly patients scheduled to undergo emergency laparotomy were offered both general anaesthesia and continuous spinal anaesthesia. Patients who chose CSA are included in this report. Data was prospectively collected over a 27 -month period at a tertiary university hospital. Ethics approval was not required as it was a prospective service evaluation. The project was registered with Clinical Audit Safety and Effectiveness (CASE 8289), University Hospitals of Leicester NHS Trust, UK. The patients provided written consent for the use of de-identified data for analysis and publication in a peer-reviewed journal. The objective was to evaluate the benefits of CSA in providing effective anaesthesia for emergency laparotomy, in enhancing recovery after major abdominal surgery and in reducing length of stay in intensive care unit.

\section{CSA Technique}

Consent: After providing detailed information regarding the technique, rationale for its use and the alternative technique (general anaesthesia) available, informed written consent was obtained. Risks detailed in the discussion and in the written consent included failure and conversion to general anaesthesia, postdural puncture headache (10\%), nerve injury with subsequent paraesthesia and or radicular leg pain, meningitis, spinal abscess and risk of loss of function of lower limbs, bowel and bladder (cauda equina syndrome; 1 in 50,000).

Insertion: Patients were anaesthetized in theatre. Insertion of a nasogastric tube was a pre-requisite prior to commencing CSA. A large bore peripheral cannula was inserted and secured. The patient was connected to standard monitoring that included ECG, pulse oximeter and non-invasive blood pressure monitor. Pre-emptive antiemetic agents were administered (ondansetron $4 \mathrm{mg}$ and dexamethasone $3.3 \mathrm{mg}$ ). A radial artery cannula was inserted in non-dominant hand to monitor invasive arterial blood pressure. Central venous catheter was inserted if indicated. The patient was connected to an infusion of metaraminol $2-5 \mathrm{mg} /$ hour prior to siting intrathecal catheter. After recording baseline haemodynamic parameter, the intrathecal catheter was inserted with patient in the sitting position. A $16 \mathrm{~g}$ Tuohy needle and 18-gauge macro catheter (Portex, Smiths Medical, Minneapolis, Minnesota, USA) was used to provide intrathecal analgesia. Following intentional dural puncture, 18 -gauge macro catheter was inserted $4-5 \mathrm{~cm}$ into the intrathecal space.

Local Anaesthetic dosage: An initial bolus of $1 \mathrm{ml}$ of $0.5 \%$ hyperbaric bupivacaine was followed by $0.5-1 \mathrm{ml}$ aliquots of 0.5 $\%$ isobaric levo-bupivacaine till the desired level of block was reached. A dense dermatomal block till thoracic T6 dermatome was achieved in all patients. Dermatomal testing was performed to fine touch and ethyl chloride spray testing for cold sensation. In patients with severe aortic stenosis or moderate-severe cardiac failure (NYHA 3 or 4), spinal anaesthesia was initiated with $0.25 \%$ isobaric levo-bupivacaine in aliquots of $0.5 \mathrm{ml}$ every 3 - 5 minutes till a dense dermatomal block (T6) was achieved.

Sedation: Oxygen was administered via a facemask. Intraoperative sedation was provided with remifentanil infusion $(0.5$ - 0.9 $\mathrm{mcg} / \mathrm{kg} /$ hour ). Tussive effect of remifentanil provided an added benefit. Spinal anaesthesia was maintained with $1-2 \mathrm{ml}$ of isobaric $0.5 \%$ levo-bupivacaine every hour. Adjuvant drugs were avoided in the intrathecal space. Patients were warned of intraoperative discomfort due to surgical handling. Intermittent bolus of propofol 10 - $20 \mathrm{mg}$ was used to enable surgical dissection in patients with adhesions, severe anxiety, history of pre-existing abdominal pain or well-developed rectus abdominis muscle.

Monitoring: Invasive monitoring included arterial blood pressure, arterial blood gas and cardiac output monitoring. Intraoperative fluids were administered based on cardiac output monitoring. At the end of the surgery, the intrathecal catheter was removed.

Post-operative analgesia: Analgesia was provided using continuous rectus sheath analgesia. A balanced postoperative analgesic regimen included regular acetaminophen $1 \mathrm{~g} 6$ hourly and oral morphine $10-15 \mathrm{mg}$ as required. Immediate postoperative care was provided in high dependency unit if appropriate. Complications to CSA recorded included paraesthesia on insertion of intrathecal catheter, difficulty in inserting the catheter, intraoperative distress, intraoperative coughing, surgical issues with the technique, nausea and vomiting, postdural puncture headache (PDPH), and any neurological deficit prior to hospital discharge. Satisfaction to the technique (excellent, good, fair and poor) was recorded. Parameters recorded include level of postoperative care required (Level 3, level 2), length of stay in intensive care unit, length of hospital stay, 30-day mortality and any complication following conversion to general anaesthesia.

\section{Results}

Adult patients undergoing emergency laparotomy were offered CSA when a physician (NG, CA, SS) proficient in the technique was on-call. After excluding coagulation dysfunction and sepsis, CSA was offered to twenty-five patients scheduled to undergo emergency laparotomy during the period of service evaluation (May 2015-Aug 2017). Four patients preferred general anaesthesia.

Twenty-one patients underwent CSA. The mean age was 75 y (range: 56 - 92 y) and gender ratio was 14: 7 (M: F). Table 1 shows co-morbidities, length of hospital stay and the mortality scores. Three patients required conversion to general anaesthesia. Reasons for failure of CSA included accidental displacement of spinal catheter in one patient and inability to thread intrathecal catheter following successful dural puncture in two patients. Following conversion to general anaesthesia and successful surgery, one patient suffered significant postoperative cognitive dysfunction resulting in pulmonary aspiration of gastric contents that necessitated mechanical ventilation and level 3 care for 7 days. The patient had pre-existing signs of early 
dementia. The length of level 2 care of the two other patients following conversion to general anaesthesia was 3 days. Eighteen patients successfully underwent emergency laparotomy under continuous spinal anaesthesia. CSA was well tolerated and there were no complications from the technique at thirty days following the surgery. None of the patients developed post dural puncture headache. Fifteen patients were successfully discharged from hospital. Three patients died during postoperative period. One patient aged 91 y died on Day 20 following worsening of pre-existing congestive cardiac failure (NYHA 4). The second patient aged 78 y died following exacerbation of pre-existing cirrhosis of liver and severe malnourishment. The third patient aged $92 \mathrm{y}$ died on day 12.

Table 1: Demographic and Outcome data of 21 patients who received continuous spinal anaesthesia.

\begin{tabular}{|c|c|c|c|c|c|c|c|c|}
\hline S No & $\begin{array}{l}\text { Age } \\
\text { (year) }\end{array}$ & ASA & $\begin{array}{c}\text { PPOSSUM } \\
\text { Mortality (\%) }\end{array}$ & Surgery & $\begin{array}{c}\text { Incision } \\
\text { Dermatome }\end{array}$ & LOS Level 2 & $\begin{array}{c}\text { LOS } \\
\text { Hospital }\end{array}$ & $\begin{array}{c}\text { 30-day } \\
\text { survival }\end{array}$ \\
\hline 1 & 85 & 3 & 18.7 & SB Resection & $\mathrm{T} 10-\mathrm{L} 1$ & 1 & 27 & Yes \\
\hline 2 & 65 & 3 & 2.9 & SB Resection & $\mathrm{T} 10-\mathrm{L} 1$ & 1 & 6 & Yes \\
\hline 3 & 58 & 4 & 15 & Ileostomy & $\mathrm{T} 10-\mathrm{L} 1$ & 0 & 6 & Yes \\
\hline 4 & 63 & 3 & 4.8 & SB Resection & $\mathrm{T} 8-\mathrm{T} 12$ & 2 & 6 & Yes \\
\hline 5 & 75 & 3 & 3.5 & SB Resection & $\mathrm{T} 10-\mathrm{L} 1$ & 0 & 14 & Yes \\
\hline 6 & 68 & 4 & 16 & Washout & $\mathrm{T} 8-\mathrm{T} 12$ & 1 & 33 & Yes \\
\hline 7 & 88 & 4 & 18.7 & SB Resection & $\mathrm{T} 9-\mathrm{T} 12$ & 1 & 12 & Yes \\
\hline 8 & 90 & 4 & 73.4 & Gastrojejunostomy & $\mathrm{T} 6-\mathrm{T} 10$ & 1 & 21 & No \\
\hline 9 & 65 & 4 & 38.2 & Adhesionolysis & $\mathrm{T} 8-\mathrm{T} 12$ & 2 & 14 & Yes \\
\hline 10 & 82 & 3 & 8.6 & Hartman's & $\mathrm{T} 7-\mathrm{L} 1$ & 0 & 25 & Yes \\
\hline 11 & 78 & 4 & 25.2 & Adhesionolysis & T10 - L1 & 1 & 7 & Yes \\
\hline 12 & 69 & 4 & 12.2 & SB Resection & $\mathrm{T} 10-\mathrm{L} 1$ & 6 & 8 & Yes \\
\hline 13 & 56 & 3 & 1.5 & Adhesionolysis & $\mathrm{T} 10-\mathrm{T} 12$ & 0 & 5 & Yes \\
\hline 14 & 83 & 3 & 9 & Enterotomy & $\mathrm{T} 8$ - T12 & 1 & 5 & Yes \\
\hline 15 & 71 & 3 & 11 & Colectomy & $\mathrm{T} 7-\mathrm{T} 12$ & 1 & 13 & Yes \\
\hline 16 & 76 & 4 & 7 & Ileostomy & $\mathrm{T} 7-\mathrm{L} 1$ & 4 & 37 & Yes \\
\hline 17 & 90 & 3 & 2.9 & SB Resection & $\mathrm{T} 12-\mathrm{L} 1$ & 1 & 3 & Yes \\
\hline 18 & 78 & 4 & 59.7 & Enterotomy & $\mathrm{T} 10-\mathrm{L} 1$ & 7 & 32 & Yes \\
\hline 19 & 91 & 3 & 17 & Hartman's & $\mathrm{T} 8-\mathrm{T} 12$ & 1 & 19 & Yes \\
\hline 20 & 72 & 4 & 50.2 & Colectomy & $\mathrm{T} 7-\mathrm{T} 12$ & 0 & 20 & No \\
\hline 21 & 92 & 4 & 34.7 & Ileostomy & $\mathrm{T} 8-\mathrm{T} 12$ & 0 & 12 & No \\
\hline
\end{tabular}

ASA: American Society of Anesthesiologists; T: Thoracic; L: Lumbar; SB: small bowel; LOS: length of stay; PPOSSUM: Portsmouth Physiological and Operative Severity Score for enumeration of Mortality and Morbidity ${ }^{[10]}$

Surgical relaxation was reported as adequate by operating surgeons in all patients. Seven patients required additional sedation with intermittent bolus of propofol for intraoperative discomfort. Reasons include anxiety (1). bowel loops adherent to the anterior abdominal wall (4) and pre-existing abdominal pain with tight rectus abdominis muscle (2). The maximum dose of propofol needed was $60 \mathrm{mg}$. There was no incidence of intraoperative nausea, vomiting or coughing. None of the patients who successfully underwent CSA required level 3 care. Mean length of stay in the high dependency unit (level 2 care) was 1.6 days. Transient paraesthesia was reported on insertion of the catheter by 12 patients. There were no neurological sequelae reported at discharge from hospital. None of the patients reported postdural puncture headache. Patient satisfaction with CSA was excellent $(76 \%)$ and good $(24 \%)$.

\section{Discussion}

CSA is an underutilized technique in modern anaesthesia practice. It involves intermittent dosing of local anaesthetic solution via an intrathecal catheter. It allows titration of the block level to the surgical requirement with significantly improved haemodynamic stability when compared to other neuraxial techniques $^{[2-4]}$. Denny et al have reported on the use of CSA in patients undergoing lower abdominal surgery ${ }^{[5]}$. Two-thirds of our cohort had the surgical incision extending above the umbilicus. Our results show that it can be used as a safe and cost-effective alternative to general anaesthesia in high-risk patients undergoing emergency laparotomy.

In our cohort, CSA provided adequate intraoperative analgesia and surgical relaxation. Intraoperative coughing and discomfort was minimized with remifentanil infusion. Nausea and vomiting during surgery was avoided by administration of pre- emptive anti-emetic agents. Intraoperative muscle relaxation was aided by small bolus of propofol $(10 \mathrm{mg})$ in patients with well-developed rectus abdominis muscle and in patients who were anxious or reported pre-existing abdominal pain. We were mindful of the risk of respiratory depression during the combined administration of propofol and remifentanil. Haemodynamic instability was minimised using an infusion of metaraminol prior to siting the intrathecal catheter. The metaraminol infusion was discontinued once sensory block regressed in 
the recovery bay. The intrathecal catheter was removed at end of the surgery and analgesia was maintained using continuous rectus sheath analgesia.

In elderly patients undergoing emergency laparotomy, general anaesthesia involves poly-pharmacy and invasive airway management. Postoperative adverse events include delirium, nausea, vomiting and there is a potential for postoperative mechanical ventilation. This can result in prolonged level 2 and often level 3 care in an intensive care unit. The average length of stay in critical care for this cohort receiving general anaesthesia for emergency laparotomy in the UK is 3 days $^{[6]}$. None of the patients who successfully underwent CSA required level 3 care. Length of stay in the high dependency unit (Level 2 care) was reduced (mean stay 1.6 days). Three patients required conversion to general anaesthesia with resultant prolonged length of stay in level 2 care (mean stay 6.6 days).

General anaesthesia can also increase the postoperative morbidity, which can result in prolonged length of hospital stay. Some of our high-risk elderly patients may not have survived a general anaesthesia and subsequent mechanical ventilation in the intensive care unit. The mean P POSSUM (Portsmouth Physiological and Operative Severity Score for enUmeration of Mortality and Morbidity) mortality score of our cohort was 20.5 $\%{ }^{[7]}$. 30-day mortality in the elderly adult undergoing emergency laparotomy is between $14.6-33.3 \%^{[8,9]}$. The 30 -day mortality in our small cohort was $14 \%(3 / 21)$.

We used a standard 16-gauge Tuohy needle for performing CSA. The advantage of using a wide bore needle and catheter is the ease of identifying the intrathecal space and subsequent insertion of the catheter ${ }^{[10]}$. However, the main concern of using a wide bore needle is the risk of developing a postdural puncture headache (PDPH). In our series, none of the patients reported PDPH. Denny et al used a 18-gauge needle and reported one case of PDPH in their series of 177 patients $^{[5]}$.

Neurological adverse effects following CSA include aseptic meningitis, spinal abscess, spinal haematoma and cauda equina syndrome. Although devastating, these adverse events are rare ${ }^{[11]}$. None of our patients reported any neurological deficit at hospital discharge. Continuous spinal anaesthesia utilizes a single drug, provides significantly improved haemodynamic control, avoids invasive airway management, enhances intraoperative analgesia and significantly reduces the need for mechanical ventilation following surgery. Post operative delirium, nausea and vomiting are minimized enhancing immediate recovery. These attributes play a significant role in avoiding level 3 care and reducing length of stay in high dependency unit. The overall benefits could result in reduced length of hospital stay and could improve 30-day mortality.

There is genuine concern of patient vomiting with a potential for pulmonary aspiration during surgery under CSA. Our practice of inserting a nasogastric tube to empty stomach contents, pre-emptive use of antiemetic agents (ondansetron and dexamethasone) and gentle surgical handling of bowel resulted in none of the patients complaining of nausea or vomiting during surgery. The risk of pulmonary aspiration in case of intraoperative vomiting is further reduced due to intact airway reflexes.

\section{Conclusion}

In conclusion, emergency laparotomy carries well-recognised intra and postoperative risks and in the elderly cohort, these risks are significant and probably life threatening[8,9]. General anaesthesia could play role in compounding these risks in the elderly patient. CSA is a well-established technique that may offer some reduction of both intra and postoperative risks with an improved immediate recovery from major surgery. Cost saving from reduced length of stay in intensive care unit following CSA is difficult to ignore.

\section{References}

1. Michaloudis, D., Fraidakis, O., Petrou, A., et al. Continuous spinal anesthesia/analgesia for perioperative management of morbidly obese patients undergoing laparotomy for gastroplastic surgery. (2000) Obes Surg 10(3): 220-229.

Pubmed | Crossref | Others

2. Denny, N.M. Continuous spinal anaesthesia and cauda equina syndrome. (1995) Anaesthesia 50: 474.

Pubmed $\mid$ Crossref $\mid$ Others

3. Jaitly, V.K., Kumar, C.M. Continuous spinal anaesthesia for laparotomy. (2009) Cur Anaesth Crit Care 20(2): 60-64.

Pubmed $\mid$ Crossref $\mid$ Others

4. Moore, J.M. Continuous Spinal Anesthesia. (2009) Am J Ther 16(4): 289-294.

Pubmed $\mid$ Crossref $\mid$ Others

5. Denny, N., Masters, R., Pearson, D., et al. Postdural puncture headache after continuous spinal anesthesia. (1987) Anesth Analg 66(8): 791-794.

Pubmed $\mid$ Crossref $\mid$ Others

6. Green, G., Shaikh, I., Fernandes, R., et al. Emergency laparotomy in octogenarians: A 5-year study of morbidity and mortality. (2013) World J Gastrointest Surg 5(7): 216-221.

Pubmed $\mid$ Crossref $\mid$ Others

7. Prytherch, D.R., Whiteley, M.S., Higgins, B., et al. POSSUM and Portsmouth POSSUM for predicting mortality. Physiological and operative severity score for the enumeration of Mortality and morbidity. (1998) Br J Surg 85(9): 1217-1220.

Pubmed | Crossref | Others

8. Howes, T.E., Cook, T.M., Corrigan, L.J., et al. Postoperative morbidity survey, mortality and length of stay following emergency laparotomy. (2015) Anaesthesia 70(9): 1020-1027.

Pubmed | Crossref | Others

9. Saunders, D.I., Murray, D., Pichel, A.C., et al. Variations in mortality after emergency laparotomy: the first report of the UK Emergency Laparotomy Network. (2012) Br J Anaesth 109(3): 368-375.

Pubmed | Crossref | Others

10. Shenkman, Z., Eidelman, L.A., Cotev, S. Continuous spinal anaesthesia using a standard epidural set for extracorporeal shockwave lithotripsy. (1997) Can J Anaesth 44(10): 1042-1046.

Pubmed | Crossref | Others

11. Horlocker, T.T., McGregor, D.G., Matsushige, D.K., et al. Neurologic complications of 603 consecutive continuous spinal anesthetics using macrocatheter and micro catheter techniques. (1997) Anesth Analg 84: 1063-1070.

Pubmed | Crossref $\mid$ Others
Ommega Online Publishers

Journal Title: Journal of Anesthesia and Surgery (JAS)

Journal Short Name: J Anesth Surg
Journal ISSN: 2377-1364

E-mail: anestheisa@ommegaonline.com

Website: www.ommegaonline.org 